\title{
SEXUALIDAD INTEGRAL AFECTIVA: UNA VISIÓN TRANSDISCIPLINARIA
}

\begin{abstract}
Albino Rojas: Universidad Pedagógica Experimental Libertador, Caracas (Venezuela)
\end{abstract}

\section{Resumen}

La sexualidad es una manifestación natural asociada al desarrollo de las dimensiones del Ser. Entendiendo que éstas se expresan a partir de los ámbitos psicológico, biológico, social, cultural y espiritual de la persona. El estudio y comprensión de esta realidad se logra, no solo con el conocimiento recíproco de los conceptos de las disciplinas que intervienen Antes, por el contrario supone, la unificación conceptual de cada una de ellas. De allí que puede hablarse de una visión integral, en la interpretación de la sexualidad. En el presente artículo se aspira establecer la manera cómo se conjugan los diferentes elementos que implican la Sexualidad para concretar una propuesta denominada: Sexualidad Integral Afectiva. Debido a la naturaleza cambiante del individuo, cada uno de ellos aborda un proceso de desarrollo. El cual será analizado desde el conocimiento de las dimensiones que lo determinan, condicionan o, en muchos casos, lo mediatizan. Intentando llegar a la unificación conceptual mencionada. Se parte de una realidad integral del ser que involucra lo bio, psico, social y espiritual, pudiéndosele agregar lo cultural (creencias y valores). Este último elemento, si bien no forma parte intrínseca del individuo, es un factor determinante en su accionar y desempeño como persona, debido a su naturaleza cambiante a lo largo de toda la historia vivida en el planeta. Esta visión transdisciplinar de la sexualidad permite una interpretación de esta manifestación del Ser implicando una reflexión para su educación.

Descriptores: Sexualidad, Integralidad, Afectividad, Transdisciplinariedad

\section{Una aproximación a la Sexualidad Integral}

La sexualidad ha sido vista, definida y enfocada desde diversas perspectivas. Entre ellas figuran la visión religiosa, ética, moral, biológica y social. De igual forma es considerada como lo permitido y lo prohibido, lo bueno y lo malo.

Gid Hoffman (2001), considera que "la depravación de las costumbres, la inmoralidad, la ausencia de valores son ahora la norma" (p.15); "Mientras el maestro trabaja para que el niño crezca sano y culto, la televisión lo bombardea con programas chabacanos, con mujeres semidesnudas de curvas generosas muy generosamente exhibidas, con personajes que hacen gala de su condición de desviados y depravados sexuales..." (p.26). "mientras el maestro trata de preparar en los niños los futuros padres y madres responsables que formen familias estables, la televisión los adoctrina en el adulterio, la infidelidad, le depravación, el divorcio, el aborto, el desprecio y el maltrato de la pareja. En la televisión aprende el niño que la 
finalidad principal del sexo es el placer y la satisfacción individual y que, para obtener ese objetivo, es lícito violar, vejar, deshonrar." (p.27)

Al referirse a internet este autor sostiene "es que la cosa no está exenta de problemas. Porque al lado de tesoros inmensos de valor incalculable, internet proporciona también el más inmundo y asqueroso estiércol que imaginarse pueda," (p.31).

Para la Organización Mundial de la Salud (OMS, 2004) conceptúa la noción de sexualidad sana o salud sexual sobre la base de tres elementos fundamentales:

1.- La aptitud para disfrutar de la actividad sexual y reproductiva, adaptándola a criterios de ética social y personal.

2.- La ausencia de miedos, sentimientos, de vergüenza, de culpabilidad, de creencias poco fundamentadas y de otros factores psicológicos que inhiban la reactividad sexual o cohíban las relaciones sexuales.

3.- La ausencia de trastornos orgánicos, enfermedades y deficiencias que dificulten la actividad sexual y reproductiva.

Por su parte Ariza, Cesari, Gabriel y Galán (1991) señalan que la sexualidad encuentra su configuración plena, en el ser humano adulto, también en tres dimensiones fundamentales:

1.- La Comunicativa, aquella que permite al ser humano ensanchar otros niveles de comunicación - como el verbal o el gestual - y llegar a la expresión corporal basada en el contacto de los cuerpos.

2.- La Experiencia del Placer, como respuesta a las sensaciones capaces de ser experimentadas como reacción a determinados estímulos.

3.- La Procreativa, en consonancia con la realidad de que es el sexo lo que divide a la humanidad en dos: hombres y mujeres.

Según la Real Academia Española (RAE, 2001), al término sexo, (Del lat. sexus) se le confieren cuatro significados: (1) Condición orgánica, masculina o femenina, de los animales y las plantas; (2) Conjunto de seres pertenecientes a un mismo sexo. Sexo masculino, femenino; (3) Órganos sexuales y (4). Placer venéreo que está obsesionado con el sexo. Nótese cómo las tres primeras aluden a una condición biológica mediante la cual se reconocen dos condiciones diferenciales entre los seres vivos: masculina y femenina. Mientras que la última se refiere a un estado anímico de goce, disfrute o satisfacción, como lo es el pacer. Haciendo la salvedad que sea de tipo venéreo. Es decir, al "deleite sexual".

Esta última dimensión del sexo es la responsable de que, alrededor de esta palabra se entrecrucen diferentes ideas, concepciones y actitudes. Muchas de ellas matizadas o mediatizadas por lo permitido o no permitido, lo moral o inmoral, lo bueno y lo malo y hasta lo natural y contranatural (o contra natura como ha llegado a considerársele), que presenta la sexualidad humana.

Para la RAE (ob. Cit), ésta se define como el "conjunto de condiciones anatómicas y fisiológicas que caracterizan a cada sexo". Sin embargo la sexualidad humana va más allá de estas condiciones, que caen en el plano de lo biológico, ya que en el hombre como ser gregario y racional se cumplen manifestaciones de conductas, 
acciones y nexos entre los dos sexos, que van a estar condicionadas por los estilos de vida del momento histórico en el que éste se desenvuelve. Aquí surge un terreno fértil para profundizar en el componente sociológico, antropológico, histórico, ético y educativo de la especie humana.

De allí entonces que, por fuerza de costumbre y tradición cuando se alude al sexo, muchas personas lo entiendan y asuman como algo que va más allá de las simples diferencias entre lo masculino y femenino y se centren en una visión estrictamente genital que le confiere al ejercicio de la función sexual única y exclusivamente la búsqueda o satisfacción del placer carnal. Dejando de lado la dimensión espiritual y de comunión de entidades que el mismo les puede brindar.

Esta visión permite afirmar que el género y el sexo deben ser entendidos como elementos socialmente construidos. En este sentido, conviene revisar algunos planteamientos que en torno a la sexualidad humana, sexo, identidad sexual, identidad de género, orientación sexual y diversidad han sido formuladas y que hacen más "excitante", esta experiencia transdisciplinar.

La sexualidad es un universo complejo en el cual intervienen aspectos tanto biológicos, como psicológicos y sociales. De igual forma engloba una serie de condiciones culturales, sociales, anatómicas, fisiológicas, emocionales, afectivas y de conducta, relacionadas con el sexo que caracterizan de manera decisiva al ser humano en todas las fases de su desarrollo.

Ciertamente se concibe como un concepto dinámico que ha ido evolucionando de la mano de la Historia del ser humano y lo acompaña desde que se nace hasta que muere. Basado en esto, la sexualidad incluye al género, las identidades de sexo y género, la orientación sexual, el erotismo, la vinculación afectiva, el amor y la reproducción. Se experimenta o se expresa en forma de pensamientos, fantasías, deseos, creencias, actitudes, valores, prácticas, roles y relaciones.

Se debe reconocer que tan relevante aspecto de la vida humana se expresa en conductas variables en las que la biología, el género y la cultura se combinan de modos diversos y no definitivos. Por lo que, en determinadas culturas o momentos geohistóricos particulares, se acepta la existencia de una diversidad sexual. En ella se presentan, además de la básica heterosexualidad, como patrón biológico para las especies, la homosexualidad, masculina o femenina, la transexualidad, la bisexualidad y la transgeneridad.

Esto conlleva un análisis desde el ejercicio de la sexualidad y de los roles de género en el devenir histórico, cultural, social, político y educativo de diversas comunidades humanas. En el contexto de esta diversidad, podría generarse un dilema de tipo religioso, familiar, político, psicológico, psiquiátrico de la actitud y comportamiento personal ante el mundo.

La diferenciación sexual presenta tres componentes: genética, hormonal y sociocultural. Corona (1994) desarrolla una teoría sobre las identidades de género, donde plantea que la misma es vista como un proceso mediante el cual los individuos desarrollan un cuerpo (anatomía), un sistema nervioso y una conducta, 
que puede ser masculina o femenina. Se inicia a nivel del código genético a nivel del gen SRY, que es el principal determinante de la diferenciación sexual masculina, tal como lo señalan Castaño, Bilbao y Urrutia (1997), y le confiere: gónadas (testículos), genitales y cerebro masculino y por ende una conducta masculina, estereotipada socialmente como tal.

Aceptar que existen diferencias entre hombres y mujeres es una forma de aceptar y entender la diversidad. En ningún momento debe utilizarse para generar parcialización o discriminación a favor de un sexo en detrimento de otro. Simplemente es una vía de autoconocimiento y de conocimiento del otro que puede resultar de ayuda al momento del ejercicio de la función sexual

Con relación al desarrollo sicológico de la sexualidad, el reconocimiento de las diferencias anatómicas sexuales ocurre entre los 3 y 5 años. La identidad de género es considerada como la "conciencia, desarrollada en la niñez temprana, entre los 3 y 6 años, de que uno es hombre o mujer". También se refiere a la "ubicación en lo femenino (feminidad), o en lo masculino (masculinidad) - sentido personal, que puede coincidir o no con el sexo biológico"

El desarrollo de la identidad de género determina la sumatoria de factores biológicos más factores socioculturales. Mientras que, los roles de género son aquellas "conductas, intereses, actitudes, habilidades y rasgos de personalidad que una cultura considera apropiados para hombres y mujeres". Hoy las sociedades occidentales tienden a ser más tolerantes e incluso permisivas.

La tipificación de género es el "proceso de socialización por el cual los niños aprenden a una edad temprana los roles de género apropiados". También se habla de estereotipos de género y se refiere a todas aquellas "generalizaciones preconcebidas acerca de la conducta del rol masculino o femenino". Por ejemplo: los varones son fuertes y las niñas débiles". Muy común en los niños y niñas en edad preescolar.

En la perspectiva psicoanalítica de la teoría freudiana la identificación es considerada como el proceso por el cual el niño pequeño adopta las características, creencias, actitudes y valores del padre del mismo sexo. En el mismo sentido la identificación sexual es el resultado de la resolución del complejo de Edipo, en el que el niño disminuye el deseo de poseer al padre del sexo opuesto y se identifica con el progenitor del mismo sexo. Esta hipótesis es influyente, pero difícil de probar.

Con relación a la perspectiva cognitiva Kohlberg (1966, citado en Psicología online, documento en línea), habla del entendimiento de género en la cual el individuo pensando activamente construye su propia tipificación de género. También plantea una constancia de género equivalente a una constancia de la categoría social vista como la "conciencia de que uno siempre será hombre o mujer". De allí que "el hecho de que un niño se percate de que su sexo siempre será el mismo conduce a la adquisición de los roles de género.

Esto ocurre en varias etapas: los niños cobran conciencia de su propio género y el de los demás; perciben que el género será el mismo a lo largo del tiempo; la niña se da cuenta de que al crecer será una mujer y el niño de que será un hombre; basan 
sus juicios de género en apariencias externas y conductas estereotipadas; entre los 3 y 7 años o después flexibilizan los juicios. En este sentido se tienen dos consideraciones: (1) Antes de que los niños alcancen la constancia de género pueden mostrar preferencias tipificadas según el género, y (2) Es posible que la conducta del género pueda sensibilizar aun más a los niños hacia la información relacionada con el género.

Dentro de la misma perspectiva cognitiva se plantea la Teoría de los Esquemas de Género de Freud (1938, citado en Psicología online, ob.cit), según la cual: "los niños se socializan en sus roles de género desarrollando una red mentalmente organizada de información sobre lo que significa ser hombre o mujer en una cultura particular". Y se desarrolla, según Lamas (2000) de la siguiente forma:

- En la infancia los niños empiezan a categorizar eventos y personas, organizando sus observaciones alrededor del esquema o categoría de género (entre 4 y 6 años)

- Una vez que saben de qué sexo son, adoptan los roles de género desarrollando un concepto de lo que significa ser hombre o mujer en su cultura

- A los 8 años los esquemas se hacen más complejos y flexibles. Por ejemplo el niño al usar un zarcillo no ve comprometido su rol de género

Esta teoría no explica por qué algunos niños muestran conductas menos estereotipadas que otros. Tampoco explica los casos donde la tipificación de género no se fortalece con un mayor conocimiento de su género. Otro de los enfoques que conviene revisar es la Teoría cognitiva social de Bandura (1986, citado en Psicología online)) según la cual "los niños aprenden los roles de género a través de la socialización. Por influencias familiares y de los pares". Aquí se podría hablar de un modelaje y Condicionamiento Operante.

En esta revisión de las diferentes dimensiones que dan forma a la sexualidad del ser humano, se tiene que la Identidad sexual se concreta en la adolescencia y se refiere a la "toma de conciencia acerca de la sexualidad". Implica reconocerse como ser sexual, identificar la propia orientación sexual, lidiar con la excitación sexual y establecer vínculos de parejas o sexuales". En este punto se habla de orientación sexual que es vista como el "foco del interés sexual y afectivo. Se presentan las variantes de: heterosexualidad, homosexualidad y bisexualidad. En este momento se pueden presentar experiencias homosexuales aisladas, atracciones o fantasías homosexuales que no determinan en modo alguno, la orientación sexual.

La homosexualidad como orientación sexual o como patrón predeterminado ha generado gran controversia. Se dice, y esto lo apoya Cornellà (2002), que puede ser determinada antes del nacimiento por influencias biológica. Se dice que si los niveles de las hormonas sexuales en un feto de cualquier sexo entre el 2 do y 5 to mes de gestación está en el rango femenino típico, es probable que la persona se sienta atraída hacia los hombres después de la pubertad. Si los niveles hormonales están en el rango masculino, es probable que la persona se sienta atraída hacia las mujeres. De igual forma hay quienes consideran que puede ser moldeada a temprana edad por factores sicosociales. 
El humor como manifestación humana mediatizada por la cultura o el momento histórico que se vive ha generado una diversa avalancha de chistes, parodias y estereotipos a favor de uno u otro sexo. En este sentido existe una amplia gama de narraciones machistas y feministas que se han ampliado hacia la diversidad 0 variabilidad sexual. De igual forma, manifestaciones culturales como la literatura, la música, la gastronomía, la publicidad, entre muchas, dan cuenta de la presencia de aspectos relacionados con la sexualidad. Sin ánimo de profundizar en los aspectos positivos o negativos que pudieran tener en el desarrollo de la misma, sólo se pretende evidenciar como no han podido escaparse de la generalización e influencia que en todos los ámbitos del quehacer humano tienen los temas relacionados con el sexo, en su visión socialmente construida.

En la cultura popular es común observar que el nombre de algunas comidas es utilizado como expresión de sexualidad. Haciendo un breve ejercicio de observación, puede realizarse una recopilación de expresiones de uso popular basadas en los alimentos de consumo generalizado, que se orientan en ese sentido. Entre ellas que destacan:

Nombres de órganos sexuales: ah buen bollo, que buen bizcocho, tremenda hallaca, comer catalina-paledonia-cuca, huevo, plátano, cambur, yuca

- Las comidas que pudieran reflejar preferencias sexuales: pártete galleta que llego tu café con leche, se partió esa galleta de soda, ser cachapera, ser un pargo

- $\quad$ Expresiones que indican acciones con contenido sexual como: Ese huevo quiere sal; Calentar la oreja; Sacarse la yuca, pelarse la yuca; Le están soplando el bistec; Ser la reina del arroz con pollo; Saber quien rellena la arepa (la canilla); Saber quien le pone el huevo a la sopa maggie; Saber quien le da gusto al huevo, No comer aguacate. Son de uso frecuente en las diferentes regiones del país

Muchas de estas expresiones son utilizadas en el galanteo, como una forma de establecer un contacto inicial con el ser objeto del deseo, mediante los llamados "piropos". Ciertamente existen diversas expresiones de este tipo que se generan en la cultura popular. La intención es evidenciar cómo los refranes como construcción literaria, utilizan elementos de la gastronomía para otorgarles una dimensión erótica y sexual. Reflejan una forma de entender en forma multidisciplinaria aspectos culturales, regionales psicológicos y sociales en el campo de la sexualidad Humana.

Lamas (1995), señala que la importancia de la literatura en la constitución de la identidad, y en concreto en la identidad de género, es manifiesta. Juega un papel crucial en la formación del imaginario por su vinculación con la fantasía; y al ser "una práctica discursiva", las normas que a través de ella se generan influyen en las personas, consciente e inconscientemente. Puede ser especialmente significativa por ejercerse en edades tempranas. Precisan la libertad para ponerse a sí mismos de múltiples formas, algunas de las cuales serán reconociblemente "femeninas", otras "masculinas". De allí que si responsabilizamos a la literatura de una pequeña parte de nuestras conductas o creencias es imposible obviar los patrones que esta alimentará y creará. 
Dado que los roles de género varían según la cultura, parece que muchas diferencias de conducta entre hombres y mujeres están causadas tanto por la socialización como por las hormonas masculinas y femeninas y otros factores congénitos ya discutidos. Aunque cada vez hay más niñas que juegan con juguetes asignados anteriormente a los chicos, lo contrario todavía es poco común.

Según Del Pino Lecuona (2000) la cultura se vale de formas simbólicas para institucionalizar la diferencia entre hombres y mujeres (en desmedro de estas últimas), separando así las prácticas, ideas y discursos que le corresponderían a cada uno. En los cuentos de Grimm y Perrault los personajes femeninos se describen en función de su aspecto y estado físico ("bonita", "delicada cosita", "enferma") como de su fuerza y estado anímico ("pobre Caperucita", "infeliz niña", "desventurada mujer", "pobre abuela") connotando en definitiva la debilidad y fragilidad del carácter de la mujer. Por el contrario, al género masculino se le reviste de un halo de perversidad y malignidad en la figura del lobo. En lo tocante a la denominación, el único rasgo destacable es la identificación de algunos protagonistas varones por su oficio de leñador o cazador, no mencionando, en ningún momento, la actividad laboral de las féminas.

Los cuentos tradicionales han sido transmitidos durante años por mujeres. Como afirma Acevedo (1991): Mientras la literatura se hallaba casi exclusivamente en manos de los hombres, eran las mujeres las que inventaban y transmitían oralmente las historias. Y añade: en los cuentos de niños y del hogar de los Hermanos Grimm se encuentran 61 personajes femeninos con poderes sobrenaturales en contraposición a 21 hombres y niños. De esta manera, unos textos con valores feministas, o con personajes femeninos con cualidades altamente positivas, eran modificados sin contemplación alguna, o en el mejor de los casos silenciados como El Príncipe Durmiente, en el que es el príncipe y no la princesa el que es rescatado por una mujer activa.

El segundo espacio de internacionalización literaria será la escuela. Niños y niñas adquirirán patrones de conducta que difícilmente tendrán modificaciones a lo largo de la vida. Para Santórum y Barrio (1998) los cuentos, las canciones y las poesías que escuché en el salón de clase, en su mayoría, llevaban una gran carga ideológica a favor de la reproducción de conductas sexistas. En algunos de ellos, no existía o era muy escasa la presencia femenina como el cuento Yo Soy Yo; en la poesía El Payaso del Viento, El Flautista de Hamelín, Pinocho, El Gato con Botas, Los dos Ratones; en las canciones Pedro comió Pan, Los Esqueletos, Que todos los Niños estén muy Atentos, El Payaso se pinchó la Nariz, Los diez Pececitos.

En otras ocasiones a las mujeres se les mencionaba en situaciones de inferioridad social o subordinación: Caperucita Roja, La Bella Durmiente, El Mono Tulín, El Conejo y la Tortuga, entre otros. Con este análisis queda manifestada la necesidad de releer críticamente los textos para formar en igualdad de sexos o más bien de géneros. En el caso de los textos literarios infantiles se han reconocido marcas explícitas que denotan líneas de comportamiento según la diferencia sexual, indicando conductas y perfiles para hombres y mujeres. a partir de estos casos se recomienda que se inserten en el abordaje transdiciplinario de la literatura como apoyo a la educación sexual. 
Otro contexto de expresión de la sexualidad lo constituye la música. La producción musical, a través de las letras de los temas musicales que se crean y difunden a toda la población no escapa de ser utilizado como vehículo de promoción de mensajes de contenido sexual. En muchos casos se potencian conductas a favor de un sexo en detrimento de otro o se promueven prácticas que dependiendo de la sociedad, son admitidos o no.

Igual consideración cabría hacerse de la publicidad. Con la idea de que "el sexo vende" se puede apreciar el elevadísimo contenido erótico y sexual que presentan la mayoría de los comerciales que se difunden en las sociedades signadas por un elevado consumismo. Muchos de estos mensajes pueden ser considerados como sexistas, promoviendo patrones a favor de uno u otro género, o de la diversidad sexual que día a día reclama más espacios de participación en lo social, político y económico y utiliza este medio de expresión como vía para difundir este mensaje.

Con relación a esto, cobra relevancia la planteado por Lamas (1996) cuando se refiere a que el género es una construcción socio - cultural, que hace referencia a la identidad de mujeres y hombres, que determina sus roles y, por tanto, cómo deben comportarse y pensarse en sociedad. Nace de la diferenciación de los sexos, como primera forma de oposición. En ello ayudan mucho las manifestaciones culturales mencionadas. El género será entonces, la consecuencia de tradiciones o costumbres transmitidas culturalmente y susceptibles de cambio mediante estrategias educativas dirigidas a individuos y colectividades.

La posición diferenciada determinada para hombres y mujeres se construye a partir de la asignación de roles, espacios, características e identidades diferentes para cada uno en razón del sexo biológico, que da como resultante una situación diferenciada- en términos de derechos, valores y oportunidades- y un código complejo que organiza y regula las relaciones entre los sexos.

La interrogante sobre la diversidad sexual puede hacerse $-y$ se ha hecho- desde la economía, la política, el psicoanálisis, la crítica literaria, la sociología, la antropología, la educación, la medicina y el derecho. Las fronteras entre los intereses, objetos de estudio y las teorías de cada disciplina son cada vez menos rígidas.

A nivel político y en el marco de los derechos humanos surge la necesidad de garantizar al individuo el desarrollo pleno de su personalidad, con sus aciertos y desaciertos y con sus gustos y preferencias. Así se consolidad las minorías, que reclaman y en muchos países se les ha concedido la posibilidad de manifestar abiertamente y en un marco de tolerancia sus preferencias sexuales.

Corona (1994), plantea que en el contexto de la visión de diversidad sexual el termino identidad sexual se vuelve un desafio para las mentalidades infantiles y adolescentes, que requieren de prescripciones externas para actuar. Lo importante de utilizar el concepto diversidad sexual es reconocer que todos los comportamientos y deseos sexuales forman parte de un amplio espectro que está disponible para el ser humano, y que todo lo que forma parte de este abanico de posibilidades sexuales tiene igual derecho de existir y manifestarse siempre que no atente contra los derechos de terceros 
Se requiere capacitar y o actualizar a los orientadores, profesores, médicos y terapeutas en una visión que complemente la formación de actitudes de respeto, empatía y congruencia con la información sobre temas biológicos como embarazo, Respuesta sexual Humana; Erotismo y Preñez y de tipo Social como Genero, Racismo, Discriminación y Homofobia entre otros.

En un estudio sobre Sexualidades diversas. Aproximaciones para su análisis, realizado en el marco del Programa universitario de estudios de género (PUEG) Salvador Cruz, (2004), plantea la necesidad de construir un lenguaje común para el entendimiento y la visibilización de las diversidades sexuales en pro de la construcción de nuevas formas de relación sin exclusión. Para ellos "sexo" debe considerarse en función de: la Sexuación, la Sexualidad y el Erotismo.

Consideran que el objetivo de la sociedad debe ser que cada cual sea feliz con su forma de expresar la sexualidad. Y esto solo será posible si consiguen despertar coherencias y evitan forzar obediencias, si se logra que el erotismo brote de cada cual y que no venga impuesto. Señalan que trabajar por el respeto a la diversidad sexual es hacerlo para que cada cual aprenda a conocerse, a aceptarse, a vivir y expresar su sexualidad de modo que sea feliz. Trabajar por la Educación Sexual es hacerlo por eliminar la concepción jerárquica de la sexualidad. Se trata de que cada cual aprenda a ser el protagonista de su propia vida sexual, a sabiendas de que se es único e irrepetible.

El tema de la sexualidad empieza a generalizarse y poco a poco se va constituyendo en un tema cotidiano de reflexión al interés por conocer los matices que puede adquirir. El trabajo desarrollado en torno a la investigación en este campo se ha dirigido hacia las identidades, las expresiones culturales, literarias, las formas de resistencias y de organización, y los estilos de vida. Esto pasa por el reconocimiento mismo de la sexualidad como una esfera de la vida independiente de la reproducción y de la presencia de otras expresiones de la sexualidad orientado hacia la no discriminación. Más aún, reta a mirar un mundo sin categorías, donde las expresiones de la sexualidad tengan cabida y sean plenamente disfrutadas, un mundo que aún ni siquiera se imaginó.

\section{Propuesta de una Visión Pedagógica concreta: La Educación Sexual Afectiva}

Como una forma de abordaje transdisciplinar de la sexualidad humana surge una propuesta metodológica basada en el interés de los participantes que serán objeto del mismo. La recomendación es que en fecha previa al evento se establezca contacto con ellos y se les solicite que de una anónima manifiesten que aspecto les gustaría fuera tratado en la charla, jornada o taller. Esto exige al organizador una preparación minuciosa de su intervención, ya que pueden surgir diversos tópicos relacionados con el tema que ellos desean conocer y que él no había considerado incluirlos.

A manera de ejemplo, como parte de la preparación de una charla dirigida a 71 estudiantes de la carrera de Educación en el Instituto Universitario Jesús Obrero de 
Fe y Alegría, ubicado en Los Flores de Catia. Antes de la actividad se les formuló la pregunta: ¿qué te gustaría saber sobre la Sexualidad?. Al analizar las respuestas llama la atención que el interés de la muestra se orientó hacia dos aspectos: el ejercicio de la función sexual y el sexo anal. En el contexto de lo que se ha discutido el segundo aspecto podría estar contenido en el primero. Lo que sucede es que los que manifestaron interés en el tema lo hicieron por curiosidad o para convencer a su pareja sexual (princialmente heterosexual) de que su práctica es común y accediera a realizarla.

Dentro del tópico Ejercicio de la función sexual los aspectos que generaron interés en el grupo fueron son muy diversos y ello obliga al conferencista a orientar su disertación o comentarios sobre esos temas que genera interés en ellos. De esta manera se garantiza que el éxito, medido en función del grado de satisfacción del participante de la charla o taller, esté garantizado. Si esta actividad se realizara en otro contexto los resultados serían diferentes.

En este mismo contexto, en el Instituto Pedagógico de Miranda "Jose Manuel Siso Martínez" se dicta el curso Puericultura Salud y Nutrición a las alumnas de la Carrera de Educación Inicial. En este curso se abordan contenidos relacionados con la Educación Integral de la Sexualidad. Esto es así, ya que corresponde tratar lo que es la Puericultura y sus dimensiones; las cuales van desde el ámbito Preconcepcional, Concepcional, De la Gestación y de la Primera y Segunda Infancia.

Los contenidos como tal responden a una propuesta de integración de diferentes disciplinas científicas que tienen injerencia en el tratamiento de los aspectos anteriormente mencionados; entre las que pueden mencionarse figuran: Medicina, Obstetricia, Pediatría, Fisiología, Sociología, Biología, Embriología, Genética, Endocrinología, entre otras. Se parte del que el ser humano debido a su capacidad de pensar y razonar, ha dedicado desde sus orígenes gran esfuerzo al cuidado de sus descendientes, consciente de lo expuestos que están a las influencia de muchos factores antes y durante la gestación, así como de la debilidad que presentan al nacer y de las dificultades que encontrarán en su adaptación a las condiciones habituales de vida.

En este contexto se presenta el curso en el entendido de que las futuras docentes de Educación Preescolar o Inicial ejercerán el despliegue de su acción pedagógica desde el nivel de maternal hasta su incorporación en la Educación Primaria, es decir, desde los 0 hasta los 6 ó 7 años. Razón por la cual requieren de una formación integral que abarque todo ese período, para comprenderlo mejor y así orientar su desempeño en función del desarrollo integral del niño o la niña que atiendan. Se le da un enfoque holístico a los tópicos relacionados con la Sexualidad.

En el Período Académico 2004 -l, correspondientes a las secciones 1, 2 y 3, lo cual daba un total de 43 alumnas. Como aporte a la visión transdisciplinaria del tema de la sexualidad, ellas discutieron y acordaron por consenso una serie de recomendaciones para desarrollar lo que se denominó una Educación Sexual Afectiva, es decir, que surja desde el amor, el afecto y los sentimientos más sublimes que deben matizar los procesos de enseñanza y aprendizaje en el marco 
de dicha integralidad del ser en crecimiento, desarrollo y formación. Generando las siguientes recomendaciones:

1.- Considerar a la Educación Integral de la sexualidad Humana, partiendo de la definición de Haffanner (1995) como "Un proceso que dura toda la vida y que está dirigido a adquirir información, formar actitudes, convicciones y valores sobre la identidad, las relaciones y la intimidad, la imagen corporal y el género sexual. En las dimensiones biológicas, socioculturales, sicológicas o sicosexuales y espirituales, desde el dominio cognitivo, afectivo y conductual, incluyendo a su vez la comunicación efectiva.

2.- Partir de que los padres y los docentes no estén bloqueados sexualmente, acepten su propia sexualidad y la de los demás y no sientan vergüenza ni culpabilidad al tratar el tema.

3.- Al enseñar educación sexual, el docente debe basarse en el respeto mutuo, comenzando por hacer énfasis en las diferencias de sexo, género e identidad sexual de cada quien.

4.- Facilitar las relaciones entre iguales, es decir, dejar que los niños y niñas jueguen, conversen y se interrelacionen.

5.- En la etapa de los 0 a os 2 años, y quizás un poco mas, evitar de provocar cambios bruscos, ya que durante estas edades es fundamental el apego afectivo y la dependencia del adulto, lo que le proporciona seguridad.

6.- El docente debe ser empático, propiciando una comunicación afectiva y afectiva, lo que va a permitir que se sienta aceptado y parte de un grupo.

7.- Se debe demostrar afecto, los besos los abrazos y las caricias son arte normal en todos los procesos del niño, adolescente y adulto, es parte de la socialización, por consiguiente, no debe existir escándalo si ocurre entre niños del mismo sexo o entre sexos opuestos.

8.- El adulto significativo debe abstenerse de escandalizarse ante las manifestaciones, conductas y curiosidad infantil, reaccionando de la mejor manera posible y preguntar con naturalidad el por qué de lo que el niño o niña hace.

9.- Antes de responder a una pregunta del niño o niña indagar el contexto en el cual surge para conocer su interés real, y al responder hacerlo con naturalidad, siempre con la verdad por delante y mostrando una actitud abierta al diálogo, donde predomine la tolerancia y el respeto a sus ideas.

10.- Como el adulto significante es un modelo a seguir, y como modelador de la conducta infantil, debe estar atento a cómo se desarrollan los procesos de imitación entre los niños y de ellos hacia el adulto.

11.- Respetar los sentimientos, observar las conductas de los niños y reforzarlas, y si no son las adecuadas o no se está de acuerdo con ellas asumir una actitud de apertura, tolerancia y afecto al intentar mediar ara generar algún cambio. En todo caso apoyarse en otros adultos significantes (pareja o docente)

12.- Evitar incurrir en los estereotipos como: los niños no lloran o las niñas solo juegan con muñecas. Los niños necesitan valorarse entre si y establecer relaciones igualitarias desde los juegos, en el uso de la ropa de variados colores, en la participación de las tareas del hogar sin distinción de sexos.

13.- Introducir conceptos como "prostitución", "homosexualidad", "transgénero", ya que muchos niños ven y oyen hablar de estos asuntos en la televisión y eso despierta su curiosidad. De esta manera se estaría educando en la no discriminación y protegiéndolo de posibles abusos o confusiones futuras. 
14.- Realizar un sondeo de opinión sobre dudas, interrogantes o temas de interés antes de realizar un abordaje con los niños y niñas sobre temas relacionados con la sexualidad humana. Muchas veces el interés de ellos es muy distinto a lo que el adulto cree.

15.- Fomentar un espíritu crítico mediante situaciones y casos concretos, considerando las consecuencias y riesgos.

16.- Fomentar el conocimiento y práctica de la higiene personal, haciendo énfasis en la zona de los genitales.

17.- Los adres deben demostrarle al niño que el llegó a sus vidas producto del amor, esta demostración se hace, no a través de las palabras, sino de los hechos propiciando en el hogar un ambiente de amor y paz, no de peleas constantes.

\section{Referencias}

ACEVEDO, R. (1991). Tareas de Investigación en el Campo de los Estudios Literarios. Cuadernos de Humanidades, Central de Publicaciones, Facultad de Humanidades USACH, Santiago

ARIZA, G., Cesari, M.D., Gabriel, M., Galan, Y. (1991). Programa integrado de pedagogía sexual en la escuela. Madrid: Narcea, S. A. de Ediciones.

CASTAÑO, L., Bilbao, J.R. y Urrutia. I. (1997). Introducción a la biología molecular y aplicación a la pediatría (5): Casos clínicos. Alteraciones genéticas en la Disgenesia. Asociaciónn Española de Pediatría;46:513-518

CIRIACO IZQUIERDO, P. (2000). Sexualidad y afectividad juvenil. Venezuela: Paulinas Distribuidoras.

CORNELLÀ, J. (2002). Identidad y orientación sexual en el niño y en el adolescente: guía para profesionales de salud. En Medwave Ene;2(1):e1212 doi: 10.5867/medwave.2002.01.1212

CORONA, E. (1994). Identidades de género: en busca de una teoría. En: Antología de la sexualidad humana.

GID HOFFMAN, J. (2001), La educación desde el banquillo de los acusados. Caracas: Sphinx

LAMAS, M. (1995). La perspectiva de género. En Revista de Educación y Cultura de la sección 47 del SNTE. http://www.latarea.com.mx/articu/articu8/lamas8.htm

LAMAS, M. (1996). El Género: una categoría útil para el análisis histórico. En: El Género: la construcción cultural de la diferencia sexual. Programa Universitario de Estudios del Género. México. Pp. 265-302.

LAMAS, M. (2000). Diferencias de sexo, género y diferencia sexual. Cuicuilco, enero-abril, año/vol. 7, número 018. Escuela Nacional de Antropología e Historia (ENAH). Distrito Federal, México 
LECUONA NARANJO, M. (2000). ¿Educamos en y para la igualdad? En Revista de la Facultad de Ciencias de la Educación, 2: 77-92, 13

Organización mundial de la salud. Salud Sexual. Organización Mundial de la Salud www.who.int/topics/sexual health/es/

Psicología online. Disponible en http://www.psicologiaonline.com/ebooks/personalidad/bandura.htm. (Consulta, enero de 2004)

Real Academia Española (RAE, 2001). Documento en línea disponible en: http://www.rae.es/recursos/diccionarios/drae

SALVADOR CRUZ, G. (2004). Sexualidades diversas. Aproximaciones para su análisis. Programa universitario de estudios de género. Universidad Nacional Autónoma de México

SANTÓRUM, R. y BARRIO, R. (1998). Del sexismo lingüístico a la diferenciación sexual en el lenguaje. Desarrollo del lenguaje y cognición. Madrid: Pirámide. 